\title{
Characterization of the ResC Sensor Kinase from Erwinia amylovora and Other Enterobacteria
}

\author{
Dongping Wang, Schuyler S. Korban, P. Lawrence Pusey, and Youfu Zhao
}

First and fourth authors: Department of Crop Sciences, and second author: Department of Natural Resources and Environmental Sciences, University of Illinois at Urbana-Champaign, Urbana 61801; and third author: United States Department of Agriculture-Agricultural Research Service, Tree Fruit Research Laboratory, Wenatchee, WA 98801.

Accepted for publication 18 January 2011.

\begin{abstract}
Wang, D., Korban, S. S., Pusey, P. L., and Zhao, Y. 2011. Characterization of the RcsC sensor kinase from Erwinia amylovora and other enterobacteria. Phytopathology 101:710-717.

$\mathrm{RcsC}$ is a hybrid sensor kinase which contains a sensor domain, a histidine kinase domain, and a receiver domain. We have previously demonstrated that, although the Erwinia amylovora $r c s C$ mutant produces more amylovoran than the wild-type (WT) strain in vitro, the mutant remains nonpathogenic on both immature pear fruit and apple plants. In this study, we have comparatively characterized the Erwinia RcsC and its homologs from various enterobacteria. Results demonstrate that expression of the Erwinia $\operatorname{rcs} C$ gene suppresses amylovoran production in various amylovoran overproducing WT and mutant strains, thus suggesting the presence of a net phosphatase activity of Erwinia RcsC.

mutant in vitro. However, virulence of the Erwinia $\operatorname{rcs} C$ mutant is partially restored by $r c s C$ homologs from Pantoea stewartii, Yersinia pestis, and Salmonella enterica but not from Escherichia coli on apple shoots. Domain-swapping experiments have indicated that replacement of the E. coli $\mathrm{RcsC}$ sensor domain by those of Erwinia and Yersinia spp. partially restores virulence of the Erwinia $\operatorname{rcs} C$ mutant, whereas chimeric constructs containing the sensor domain of $E$. coli RcsC could not rescue virulence of the Erwinia $r c s C$ mutant on apple. Interestingly, only chimeric constructs containing the histidine kinase and receiver domains of Erwinia RcsC are fully capable of rescuing amylovoran production. These results suggest that the sensor domain of RcsC may be important in regulating bacterial virulence, whereas the activity of the histidine kinase and receiver domains of Erwinia $\mathrm{RcsC}$ may be essential for amylovoran production in vitro.
\end{abstract} Findings have also demonstrated that $r c s C$ homologs from other enterobacteria could not rescue amylovoran production of the Erwinia $\operatorname{rcs} C$

Erwinia amylovora, the causal agent of fire blight on apple and pear, is a member of the family Enterobacteriaceae, which also includes the genera Escherichia, Pantoea, Salmonella, and Yersinia. As one of the main virulence factors, E. amylovora produces the capsular exopolysaccharide (EPS) amylovoran to cause disease $(3,29)$. Amylovoran is an acidic heteropolymer composed of a branched repeating unit consisting of galactose, glucose, and pyruvate residues (19). Mutant strains deficient in amylovoran production are nonpathogenic (23). It has been reported that amylovoran has multiple functions, including plugging plant vascular tissues, movement through cortical tissue, retention of water and nutrients, and biofilm formation $(16,21,22)$.

In E. amylovora, two-component signal transduction systems play critical roles in sensing of and response to environmental conditions to regulate amylovoran production and virulence (31). Among them, the Rcs phosphorelay system, including RcsBCD, is essential for virulence, and it positively regulates amylovoran production (23). The membrane-localized $\mathrm{RcsC}$ is a hybrid sensor kinase composed of a sensor (input), a histidine kinase (transmitter), and a receiver (output) domain. The sensor domain contains a periplasmic region flanked by either one or two transmembrane (TM) fragments (Supplemental Figure 1). Although the exact signal for $\mathrm{RcsC}$ is unclear, genetic analyses suggest that,

Corresponding author: Y. F. Zhao; E-mail address: zhao888@illinois.edu

* The $\boldsymbol{e}$-Xtra logo stands for "electronic extra" and indicates that the online version contains one supplemental figure. Figure 3 appears in color online.

doi:10.1094/PHYTO-09-10-0258

(c) 2011 The American Phytopathological Society
Additional keywords: fire blight, polysaccharide, regulation.

upon stimulation, RcsC autophosphorylates on a conserved histidine residue in the histidine kinase domain. Subsequently, the phosphoryl group is transferred to an aspartate residue in the receiver domain which, in turn, activates the whole Rcs phosphorelay system through RcsD, another membrane-localized protein, and $\mathrm{RcsB}$, the response regulator $(15,17,26)$. Upon phosphorylation, phospho-RcsB binds to DNA and regulates gene expression. Phospho-RcsB can also be dephosphorylated by RcsC and RcsD in reverse reactions (13).

The Rcs phosphorelay system is first identified by its role in transcriptional regulation of genes for capsular polysaccharide in Escherichia coli (17). Later studies have demonstrated that the Rcs system is involved in regulation of a wide array of phenotypes such as swarming motility, antibiotic resistance, and biofilm formation $(8,12,25)$. In Salmonella enterica and Yersinia pseudotuberculosis, the Rcs system positively regulates multiple virulence factors, and $r c s$ mutants are highly impaired in their ability to colonize host cells $(6,7,11)$. In Pantoea stewartii, the Rcs phosphorelay system also plays a major role in regulating bacterial virulence (18). Previously, we have demonstrated that the Rcs system in E. amylovora is essential for regulating virulence (23). The $r \operatorname{cs} B$ and $r c s D$ mutants are deficient in amylovoran production and cannot cause disease on host plants. Although the $r c s C$ mutant produces a high level of amylovoran in vitro, it remains nonpathogenic on host plants (23).

It has been reported that $\mathrm{RcsC}$ has both kinase and phosphatase activities in E. coli $(5,17)$. In E. coli, loss of the RcsC function results in phenotypes with increased mucoidy and decreased motility; this is due, in part, to elevated levels of phospho-RcsB, indicating a net phosphatase activity of $\mathrm{RcsC}$ (9). The net direction of the flow of phosphoryl groups and strength of the 
output from this signaling pathway is modulated by different environmental signals as well as other periplasmic proteins such as DjlA (5). Recently, comparative functional studies have shown that biofilm formation in the $E$. coli $\mathrm{K}-12 \operatorname{rcs} C$ mutant can be rescued by the RcsC homolog from $S$. enterica but not from $Y$. pestis (13). Furthermore, a chimeric protein composed of the sensor domain of E. coli $\mathrm{K}-12 \mathrm{RcsC}$ and the histidine kinase and receiver domains of $Y$. pestis is capable of fully restoring biofilm formation, thus suggesting a different net kinase activity for RcsC of various sources (i.e., different kinase/phophatase ratio) (13). These results suggest that there is a link between the $\mathrm{RcsC}$ sensor domain and biofilm formation in $E$. coli.

In this study, we comparatively characterized $\operatorname{rcs} C$ homologs from five bacterial species within the family Enterobacteriaceae. Comparative analyses showed that the nonpathogenic phenotype of $E$. amylovora $r c s C$ mutant could not be complemented by $r c s C$ homologs from Escherichia coli but it could be partially restored by those from $P$. stewartii, $Y$. pestis, and S. enterica. Further analyses showed that two chimeric constructs containing sensor domains of either Erwinia or Yersinia RcsC and histidine kinase and receiver domains of $E$. coli $\mathrm{RcsC}$ partially restored virulence of the Erwinia $r c s C$ mutant, whereas chimeric constructs containing the sensor domain of $E$. coli and histidine kinase and receiver domains of Yersinia spp. could not rescue bacterial virulence and growth. In addition, amylovoran production in Erwinia $\operatorname{rcs} C$ was not restored by all other ResC homologs and chimeric proteins, except for the chimeric construct containing the histidine kinase and receiver domains of Erwinia RcsC.

\section{MATERIALS AND METHODS}

Bacterial strains and culture media. Bacterial strains and plasmids used in this study are listed in Table 1. The LuriaBertani (LB) medium was routinely used for culturing $E$. amylovora. When necessary, the following antibiotics were added to the medium: kanamycin at $50 \mu \mathrm{g} \mathrm{ml}^{-1}$ and ampicillin at $100 \mu \mathrm{g}$ $\mathrm{ml}^{-1}$. Amylovoran production was determined by growing bacterial cells in MBMA medium (3 g of $\mathrm{KH}_{2} \mathrm{PO}_{4}, 7 \mathrm{~g}$ of $\mathrm{K}_{2} \mathrm{HPO}_{4}$, $1 \mathrm{~g}$ of $\left[\mathrm{NH}_{4}\right]_{2} \mathrm{SO}_{4}, 2 \mathrm{ml}$ of glycerol, $0.5 \mathrm{~g}$ of citric acid, and $0.03 \mathrm{~g}$ of $\mathrm{MgSO}_{4}$ ) containing $1 \%$ sorbitol (2).

DNA manipulation and sequence analysis. Plasmid DNA purification, polymerase chain reaction (PCR) amplification of genes, isolation of fragments from agarose gels, cloning, restriction enzyme digestion, and T4 DNA ligation were performed using standard molecular procedures (20). DNA sequencing was performed at the W. M. Keck Center for Functional and Comparative Genomics at the University of Illinois at Urbana-Champaign. Sequence alignment and contig assembly were conducted using Sequencher 4.7 software. Database searches were conducted using the BLAST programs at the National Center for Biotechnology Information (www.ncbi.nlm.nih.gov/BLAST) (1).

Cetylpyrimidinium chloride assay for determining amylovoran concentration. The amylovoran concentration in supernatants of bacterial cultures was quantitatively determined by a turbidity assay with cetylpyrimidinium chloride (CPC) as previously described $(23,24,31)$. Briefly, bacterial suspensions were grown overnight in LB broth with or without appropriate anti-

TABLE 1. Bacterial strains, plasmids, and primers used in this study

\begin{tabular}{|c|c|c|}
\hline Strains, plasmids, or primers & Relevant characters or sequences $\left(5^{\prime}-3^{\prime}\right)^{\mathrm{a}}$ & Reference or source \\
\hline \multicolumn{3}{|l|}{ Erwinia amylovora } \\
\hline Ea1189 & Wild type, isolated from apple & 4 \\
\hline $\mathrm{Ea} 273$ & Wild type, isolated from apple & 24 \\
\hline Z0271 $\Delta o m p R$ & $\mathrm{Km}^{\mathrm{R}}$-insertional mutant of $o m p R$ of Ea1189, $\mathrm{Km}^{\mathrm{R}}$ & 31 \\
\hline Z0270_envZ & $\mathrm{Km}^{\mathrm{R}}$-insertional mutant of envZ of Ea1189, $\mathrm{Km}^{\mathrm{R}}$ & 31 \\
\hline \multicolumn{3}{|l|}{ Z0270-71 } \\
\hline$\Delta e n v Z / o m p R$ & $\mathrm{Km}^{\mathrm{R}}$-insertional mutant of envZ-ompR operon of Ea1 $189, \mathrm{Km}^{\mathrm{R}}$ & 31 \\
\hline Z2797هhns & $\mathrm{Km}^{\mathrm{R}}$-insertional mutant of hns of Ea1189, $\mathrm{Km}^{\mathrm{R}}$ & This study \\
\hline \multicolumn{3}{|l|}{ Escherichia coli } \\
\hline DH10B & $\begin{array}{l}\mathrm{F}^{-} \text {mcrA } \Delta(\text { mrr-hsdRMS-mcrBC) } \text { (R80lacZ } \Delta \mathrm{M} 15 \Delta \text { lacX74 rec } \mathrm{A} 1 \text { end } \mathrm{A} 1 \text { ara } \Delta 139 \\
\quad \Delta(\text { ara, leu }) 7697 \text { galU galK } \lambda \text {-rpsL }\left(\mathrm{Str}^{\mathrm{R}}\right) \text { nup } \mathrm{G}\end{array}$ & Invitrogen \\
\hline \multicolumn{3}{|l|}{ Plasmids } \\
\hline pGEM T-easy & $A p^{R}$, polymerase chain reaction $(\mathrm{PCR})$ cloning vector & Promega Corp. \\
\hline pWDP2 & 3.88 -kb PCR fragment containing $r c s C_{E a}$ gene in pGEM T-easy vector & 23 \\
\hline pBMM631 & $\operatorname{rcs} C_{Y p-E c}$ in $\mathrm{pBMM} 101$ & 13 \\
\hline pBMM639 & $r c s C_{E c-Y p}$ in $\mathrm{pBMM} 101$ & 13 \\
\hline pWDP777 & $r c s C_{E a}$ in $\mathrm{pBMM} 101$ & This study \\
\hline pWDP778 & $r c s C_{Y p}$ in $\mathrm{pBMM} 101$ & This study \\
\hline pWDP781 & $r c s C_{S e}$ in $\mathrm{pBMM} 101$ & This study \\
\hline pWDP779 & $r c s C_{E a-E c}$ in $\mathrm{pBMM} 101$ & This study \\
\hline pWDP780 & $r c s C_{E c-E a}$ in $\mathrm{pBMM} 101$ & This study \\
\hline \multicolumn{3}{|l|}{ Primers $^{\mathrm{b}}$} \\
\hline Ea1 & TCGAGCCATGGCCGGGATAACTGGCTTTTTCT (NcoI) & $\ldots$ \\
\hline $\mathrm{Ea} 2$ & 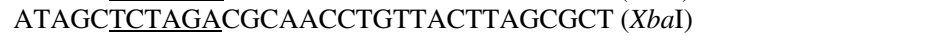 & $\ldots$ \\
\hline Ea3 & CAGAAACATCGATTTAGACTGGCTTGC ( $\mathrm{ClaI})$ & $\ldots$ \\
\hline $\mathrm{Ea} 4$ & AAACATCGATGTTCCTGGCGACCGTTA (ClaI) & $\ldots$ \\
\hline Yp1 & TCGAGCCATGGTTTTTCATGGTATCCGATTG (NcoI) & $\ldots$ \\
\hline Yp2 & ATAGCTCTAGACGTGATCGCCGTACTTGGTC (XbaI) & $\ldots$ \\
\hline
\end{tabular}

${ }^{a} \mathrm{Km}^{\mathrm{R}}$ and $\mathrm{Ap}^{\mathrm{R}}=$ kanamycin and ampicillin resistance, respectively.

${ }^{\mathrm{b}}$ Underlined nucleotides are restriction sites added and the restriction enzymes are indicated at the end of primers. 
biotics, harvested by centrifugation, and washed three times with phosphate-buffered saline (PBS). After the final wash, pelleted bacterial cells were resuspended in $200 \mu \mathrm{l}$ of PBS, and $100 \mu \mathrm{l}$ of bacterial suspension was added to $10 \mathrm{ml}$ of MBMA medium with $1 \%$ sorbitol. Following 2 days of incubation at $28^{\circ} \mathrm{C}$ with shaking, bacterial cells were pelleted by centrifugation, and $50 \mu \mathrm{l}$ of CPC (50 mg ml${ }^{-1}$ ) was added to $1 \mathrm{ml}$ of supernatant. After $10 \mathrm{~min}$ of incubation at room temperature, amylovoran concentration was determined by measuring optical density at $600 \mathrm{~nm}\left(\mathrm{OD}_{600}\right)$ turbidity. The final concentration of amylovoran production was normalized for a cell density of 1.0. For each strain tested, the experiment was repeated at least three times.

Cloning $r c s C$ genes from $E$. amylovora, $S$. enterica, $Y$. pestis, and $\boldsymbol{P}$. stewartii. The $\operatorname{rcs} C$ genes from $E$. amylovora $\left(r c s C_{E a}\right), S$. enterica serovar Typhimurium LT2 $\left(r c s C_{S e}\right)$, and $Y$. pestis $\left(r c s C_{Y p}\right)$ were cloned into pBMM101 vector under the control of the ptrc promoter (5). Briefly, genes were amplified from genomic DNA using high-fidelity platinum $P f u$ DNA polymerase (Invitrogen) and the following primer pairs: Ea1 and Ea2 for $r c s C_{E a}, \mathrm{Se} 1$ and $\mathrm{Se} 2$ for $r c s C_{S e}$, and Yp1 and Yp2 for $r c s C_{Y p}$. PCR products were digested with $N c o \mathrm{I}$ and $\mathrm{Xba \textrm {I }}$ and ligated with the $N c o \mathrm{I} / \mathrm{Xba \textrm {I }}$ digested vector, resulting in plasmids pWDP777 $\left(r c s C_{E a}\right)$, pWDP778 $\left(r c s C_{Y p}\right)$, and pWDP781 $\left(r c s C_{S e}\right)$ (Fig. 1). For cloning the $r c s C$ gene from $P$. stewartii, the primer pair Ps1-Ps2, without restriction sites, was used to amplify $3.41-\mathrm{kb}$ DNA fragments from the $P$. stewartii strain containing upstream and downstream sequences of the $r c s C$ gene. The PCR fragment was cloned into the pGEM T-easy vector through A-T ligation. The final plasmids were designated as pWDP782. All plasmids were introduced into E. coli DH10B strain by electroporation. Transformants were selected on LB plates supplemented with ampicillin. Their genotypes were confirmed by enzymatic digestion and sequencing.

Construction of chimeric $r c s C$ constructs. A $1.4-\mathrm{kb}$ DNA fragment encoding the $\mathrm{N}$-terminal region of $r c s C_{E a}$ was amplified using primers Ea1 and Ea3. The Ea3 primer introduced a ClaI site into the $\operatorname{rcs} C_{E a}$ sequence and the fragment was digested with $N c o$ I and ClaI. This ClaI site occurs naturally in the $\operatorname{rcs}_{E c}$ allele. Plasmid pBMM102, encoding $r c s C_{E c}$, was digested with $\mathrm{NcoI} / \mathrm{Cla} \mathrm{I}$ and ligated with the digested $\mathrm{N}$-terminal region of $r c s C_{E a}$ product, resulting in plasmid pWDP779. Similarly, primers $\mathrm{Ea} 2$ and Ea4 were used to amplify a DNA fragment coding for the C-terminal end of $r c s C_{E a}$, while also introducing a ClaI site at the 5 ' end of the fragment. This DNA fragment and pBMM102 were digested with $C l a \mathrm{I}$ and $\mathrm{XbaI}$ and ligated, thus resulting in plasmid pWDP780. The protein sequence around the ClaI site (SKSM) in the histidine kinase domain is identical among Erwinia and Yersinia spp. and E. coli. All plasmids are sequenced to confirm integrity of the inserts (Fig. 1). Chimeric constructs for $r c s C_{Y p-E c}$ (pBMM631) and $r c s C_{E c-Y p}$ (pBMM639) have been generously provided by Dr. David Clarke (13).

Virulence assays on apple plants and immature pear fruit. Virulence assays were performed using young annual shoots of 'Gala' apple, 22 to $25 \mathrm{~cm}$ in length, by pricking the tip with a needle and pipetting $5 \mu$ of pathogen suspension $\left(\mathrm{OD}_{600}=0.1\right)$ onto the wounded tissue. For each bacterial strain, six to seven shoots were inoculated. Plants were kept in a greenhouse at $25^{\circ} \mathrm{C}$ and a 16-h light photoperiod, and periodically evaluated for disease development for up to 8 days following inoculation by measuring length of the necrotic tissue. The experiment was performed at least three times.

Virulence and bacterial growth assays were performed using immature pear fruit as previously described $(27,28,30)$. Wounded fruit was inoculated with $2 \mu \mathrm{l}$ of cell suspension $\left(\mathrm{OD}_{600}=0.001\right)$ and incubated in a controlled humidity chamber at $26^{\circ} \mathrm{C}$. Fruit tissues surrounding the inoculation site were excised using a no. 4 cork borer as described previously $(27,28)$, and homogenized in $0.5 \mathrm{ml}$ of $0.5 \times$ PBS. Bacterial growth was monitored by dilution plating on LB medium amended with appropriate antibiotics. For

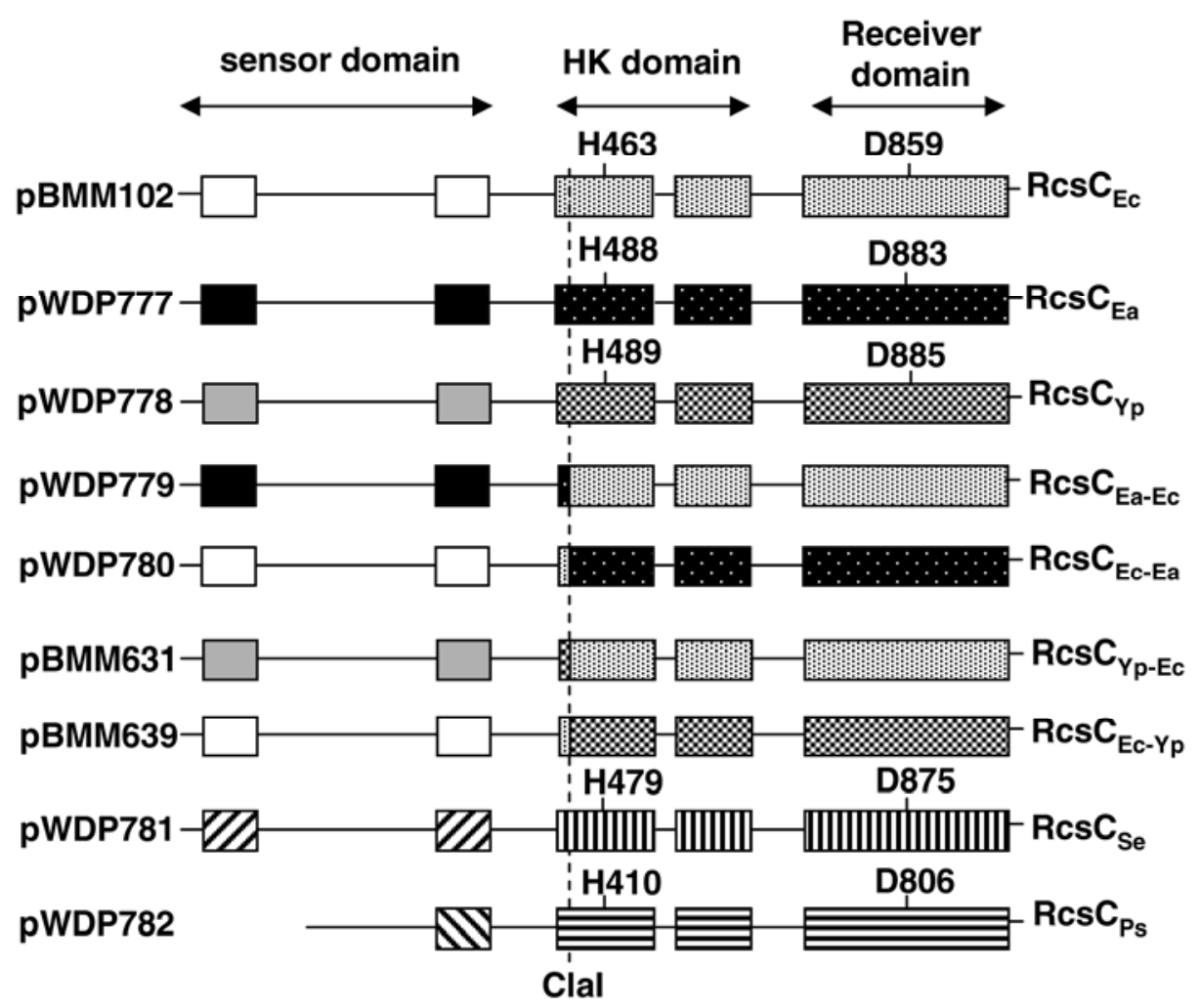

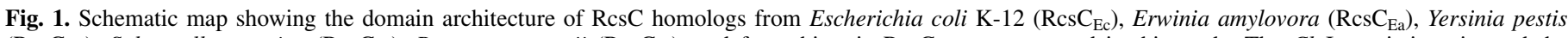

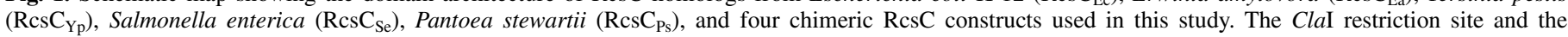

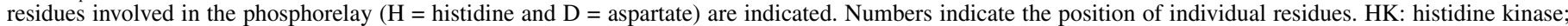

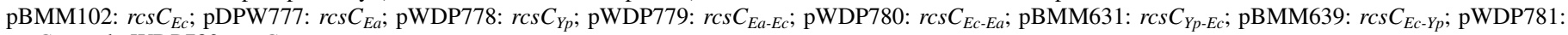
$\operatorname{rcs}_{S e}$; and pWDP782: $r \operatorname{cs} C_{P s}$. 
each strain tested, fruit were assayed in triplicate, and each experiment was performed at least three times.

\section{RESULTS}

Comparative in silico analysis of $\mathrm{RcsC}$ from enterobacterial species. The predicted amino acid lengths of RcsC are $880(P$. stewartii), 933 (E. coli), 948 (S. enterica), 957 (Y. pestis), and 987 (E. amylovora). As predicted by the SMART program, RcsC homologs contain two TM domains, except for P. stewartii RcsC, which has a single TM domain (Supplemental Figure S1). Multiple sequence alignment analysis of RcsC homologs has revealed that length variations are mainly due to variations in amino acid residues in sensor domains. A BLAST search has revealed that E. amylovora $\mathrm{RcsC}$ shares 60, 61, 57, and $66 \%$ sequence identities and $75,75,73$, and $80 \%$ sequence similarities with those of E. coli, S. enterica, Y. pestis, and $P$. stewartii, respectively. The sensor domain of E. amylovora $\mathrm{RcsC}$ shares 41, 43, 39, and 43\% sequence identities and 63, 64, 64, and 67\% sequence similarities with those of E. coli, S. enterica, $Y$. pestis, and $P$. stewartii, respectively. Moreover, the histidine kinase and receiver domain of E. amylovora $\mathrm{RcsC}$ share 68, 68, 65, and 73\% sequence identities and $80,79,75$, and $84 \%$ sequence similarities with those of E. coli, S. enterica, Y. pestis, and P. stewartii, respectively. These analyses suggest that the histidine kinase and receiver domain of the $\mathrm{RcsC}$ sensor kinase is more conserved than the sensor domain in terms of sequence similarities among the five enteric bacteria.

ResC suppresses amylovoran production in vitro. Previously, we reported that $\mathrm{RcsC}$ negatively regulated amylovoran production in vitro (23). The $r c s C$ mutant produced approximately sevenfold more amylovoran compared with that of the wild type (WT) at $48 \mathrm{~h}$. A similar effect was observed for $r \operatorname{cs} C_{E a}$ cloned in pBMM101 and pGEM T-easy vectors; both partially restored the amylovoran production phenotype of the mutant (Fig. 2B, Ea1 and Ea2). The complemented strains produced less than half of the amylovoran compared with that of the $r c s C$ mutant (23). When RcsC was overexpressed in other amylovoran overproducing WTs or mutants, decreased amylovoran production by $\geq 50 \%$ in both WT or mutant strains was observed (Fig. 3A). These results indicated that $r \operatorname{cs} C_{E a}$ acted as a negative regulator of amylovoran production in vitro, suggesting that it possessed a net phosphatase activity.

All ResC but a single chimeric construct could not restore the amylovoran production phenotype in the $r \operatorname{cs} C$ mutant. To determine whether amylovoran biosynthesis could be complemented by different RcsC homologs and chimeric proteins, bacterial strains were grown in MBMA medium for $48 \mathrm{~h}$ and the amylovoran level was quantified by a CPC turbidity assay as described above. In this study, all $\operatorname{rcs} C$ homologs were not capable of complementing amylovoran production in the $\operatorname{rcs} C$ mutant; instead, these homolog-complemented strains produced higher levels of amylovoran than those of the $r \operatorname{cs} C$ mutant (Fig. $3 \mathrm{~B})$. These results indicated that RcsC homologs from other enterobacteria could not complement the amylovoran production phenotype in vitro, suggesting that these homologs might yield net kinase activities in E. amylovora.

Amylovoran production of the $\operatorname{rcs} C$ mutant complemented by chimeric constructs was also determined (Fig. 3C). Complemented strains containing $r c s C_{Y p-E c}, r c s C_{E c-Y p}$, and $r c s C_{E a-E c}$ produced a higher level of amylovoran than that of $r c s C$ mutant. In contrast, $r c s C_{E c-E a}$ was able to rescue amylovoran production, and the complemented strain produced an amount of amylovoran equivalent to that of the strain expressing $r c s C_{E a}$. These results indicated that the histidine kinase and receiver domains of the Erwinia $\mathrm{RcsC}$ are essential for amylovoran production in vitro.

All but an $\mathrm{ResC}_{E c}$ homolog could partially rescue virulence of the $r \operatorname{cs} C$ mutant in vivo. Previously, we demonstrated that
$\mathrm{RcsC}$ was required for virulence in E. amylovora (23). To test whether different RcsC homologs could rescue virulence, the virulence of the $\operatorname{rcs} C$ mutant complemented with different homolog constructs was tested on Gala apple shoots. Disease severity was periodically measured for up to 8 days as the length of symptom development from the original inoculated site. The WT strain caused visible necrosis around the inoculated site 2 days post inoculation (dpi). The disease rapidly progressed along the shoot, with a mean rate of $\approx 2.75 \mathrm{~cm} /$ day and covered $23.0 \mathrm{~cm}$ at 8 dpi (Figs. 2 and 4A). As expected, the $\operatorname{rcs} C$ mutant and $r c s C$ mutant containing the vector plasmid pBMM101 did not cause

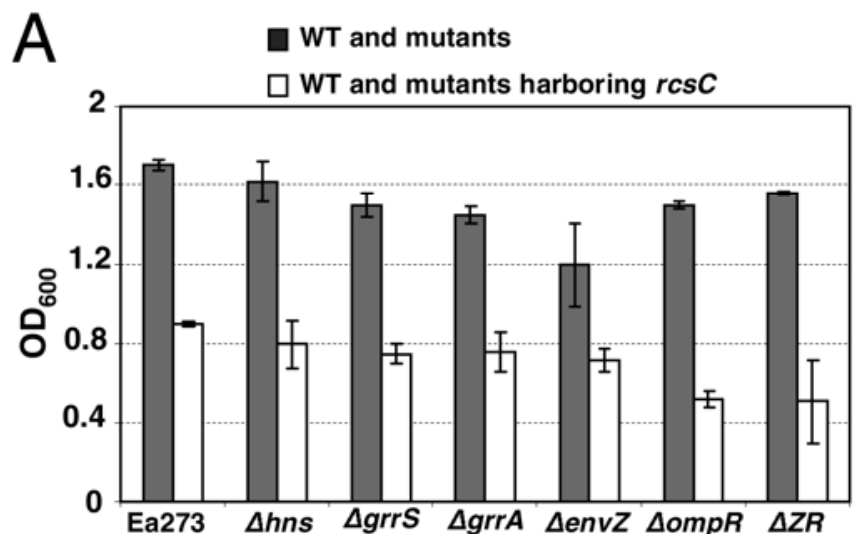

B
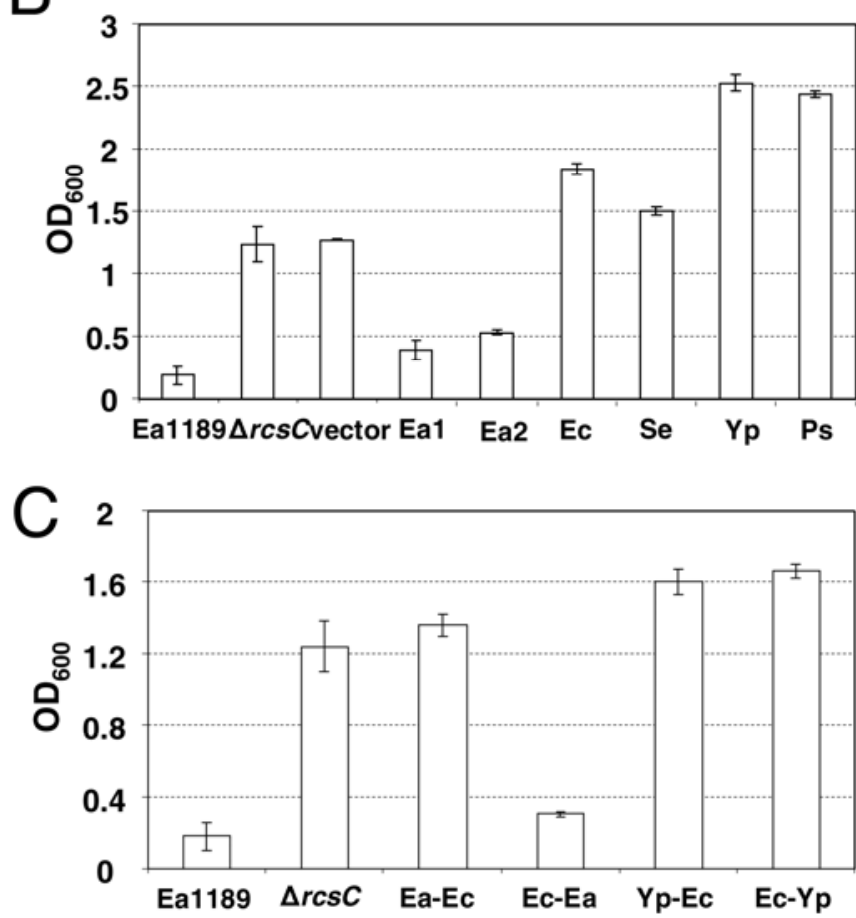

Fig. 2. Amylovoran production of bacterial strains in vitro. A, Amylovoran production of the wild-type (WT) and mutant strains with or without expressing $r c s C_{E a}$ (pWDP2 or pWDP777). B, Amylovoran production of WT, $r c s C$ mutant, and complementation strains. Vector: $\triangle r c s C$ (vector pBMM101); Ea1: $\Delta r c s C$ ( $r c s C_{E a}$, pWDP2); Ea2: $\Delta r c s C$ ( $r c s C_{E a}$ pWDP777); Ec: $\Delta r c s C$ $\left(r c s C_{E c}\right.$ pBMM102); Se: $\Delta r c s C$ ( $r c s C_{S e}$ pWDP781); Yp: $\Delta r c s C \quad\left(r c s C_{Y p}\right.$ pWDP778) and Ps: $\Delta r c s C\left(\operatorname{rcs} C_{P s}\right.$ pWDP782). C, Amylovoran production of WT, $r c s C$ mutant and $r c s C$ mutant harboring chimeric constructs. Ea-Ec: $\Delta r c s C$ ( $r c s C_{E a-E c}$ pWDP779); Ec-Ea: $\Delta r c s C$ ( $r c s C_{E c-E a}$ pWDP780); Yp-Ec: $\Delta r c s C$ ( $r c s C_{Y p-E c}$ pBMM631); and Ec-Yp: $\Delta r c s C\left(r c s C_{E c-Y p}\right.$ pBMM639). Bacterial strains were grown in MBMA media with $1 \%$ sorbitol for $48 \mathrm{~h}$ at $28^{\circ} \mathrm{C}$ with shaking. The amount of amylovoran was measured with the cetylpyrimidinium chloride assay and normalized to a cell density of 1 . Data points represent the means of three replicates \pm standard deviations. Similar results were obtained in three independent experiments. $\mathrm{OD}_{600}=$ optical density at $600 \mathrm{~nm}$. 
disease (Fig. 2). Both $r c s C_{E a}$ constructs fully complemented virulence of the mutant on Gala shoots as expected, and mean lengths of diseased tissues were 23.5 and $22.0 \mathrm{~cm}$ at 8 dpi (Figs. 2 and 4A; data not shown). Other homologs, including $r c s C_{Y p}$, $r c s C_{P s}$, and $r c s C_{S e}$, also promoted disease development at varying levels but they were not as effective as $\operatorname{rcs} C_{E a}$. However, the $r c s C_{E c}$ did not restore disease formation in the $r c s C$ mutant, suggesting that it could not complement the mutant (Figs. 2 and 4A).

The sensor domain of $\operatorname{ResC}_{E c}$ affects its ability to complement the $\boldsymbol{r c s} C$ mutant. To better understand why $\mathrm{RcsC}_{E c}$ cannot complement the virulence of the $r c s C$ mutant, we constructed several chimeric constructs (Fig. 1). To test whether chimeric $r c s C$ constructs could complement the $r \operatorname{cs} C$ mutant, a virulence test was performed for the $\operatorname{rcs} C$ mutant harboring different chimeric constructs on Gala plants, and disease severity was determined as described above. Replacement of the sensor domain of $r c s C_{E c}$ with that of either $r c s C_{E a}$ or $r c s C_{Y p}$ restored disease formation, with lengths of diseased tissues reaching 18.8 and $4.2 \mathrm{~cm}$, respectively (Figs. 2 and 4B). In contrast, when the sensor domain of $r c s C_{E a}$ and $r c s C_{Y p}$ was replaced with that of $r c s C_{E c}$, the ability to complement the $r c s C$ mutant to cause disease was completely eliminated (Figs. 2 and 4B). These results indicated that the sensor domain of $\mathrm{RcsC}$ might play an important role in the virulence of E. amylovora.
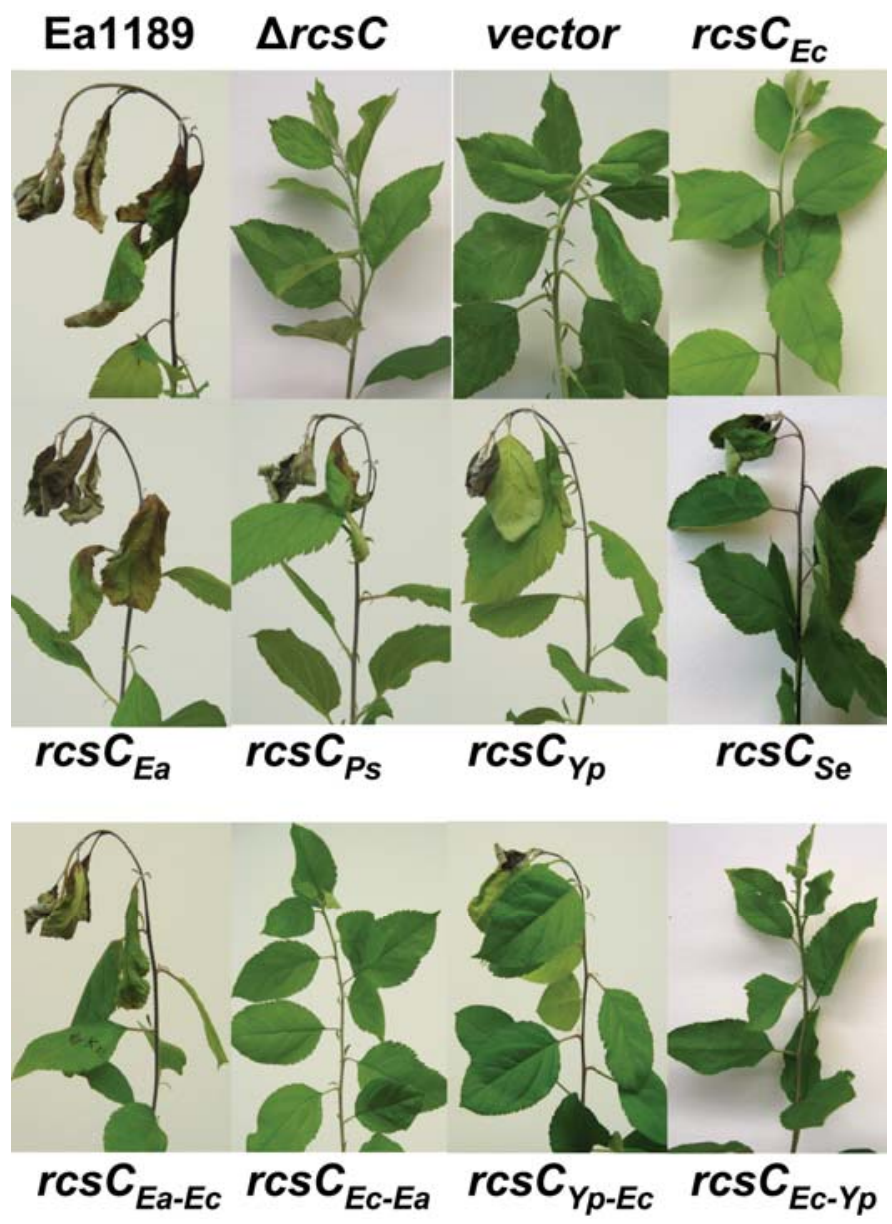

Fig. 3. Virulence tests of Erwinia amylovora wild type (WT), $r c s C$ mutant, and $r c s C$ mutant harboring different $r c s C$ constructs on 'Gala' apple shoots. Vector: $\Delta r c s C$ (vector, pBMM101); $r \operatorname{cs} C_{E a}: \Delta r c s C$ ( $r c s C_{E a}$ pDPW777); $r c s C_{E c}$ : $\Delta r c s C\left(r c s C_{E c}\right.$ pBMM102); $r c s C_{S e}: \Delta r c s C\left(r c s C_{S e}\right.$ pWDP781); $r c s C_{\mathrm{Ps}}: \Delta r c s C$ $\left(r c s C_{P s}\right.$ pWDP782) and $r c s C_{Y p}: \Delta r c s C$ (rcs $C_{Y p}$ pWDP778), $r c s C_{E a-E c}: \Delta r c s C$ $\left(r c s C_{E a-E c}\right.$ pWDP779), $\quad r c s C_{E c-E a}: \Delta r c s C$ (rcs $C_{E c-E a}$ pWDP780), $r c s C_{Y p-E c}$ :

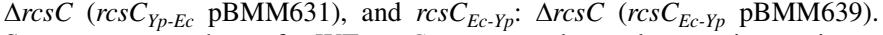
Symptoms were shown for WT, $r c s C$ mutant, and complementation strains at 8 days postinoculation.
Impact of ResC homologs and chimeric constructs on bacterial growth on immature pear fruit. Symptom development and bacterial growth were also monitored on immature pear fruit for the $r \operatorname{cs} C$ mutant complemented by various homologs and chimeric constructs. As expected, the $r c s C$ mutant complemented by $\operatorname{rcs} C_{E a}$ fully restored its virulence and bacterial growth (Fig. 5A; data not shown). RcsC homologs from Yersinia and Pantoea spp. were also capable of fully rescuing bacterial growth on immature pear fruit (Fig. 5A). However, symptom development for $r c s C_{S e}$ was slightly delayed and bacterial population size was reduced by $\approx 10$-fold at 2 and 3 dpi (Fig. 5A; data not shown). Furthermore, E. coli RcsC was not capable of supporting growth of the $\operatorname{rcs} C$ mutant (Fig. 5A). Survival of the $\operatorname{rcs} C$ mutant containing $r c s C_{E c}$ was similar to that of the $\operatorname{rcs} C$ mutant or with the empty vector (data not shown).

Growth of the $r c s C$ mutant complemented with RcsC chimeric constructs was also monitored. The $\operatorname{rcs} C_{E a-E c}$ fully restored the ability of the $\operatorname{rcs} C$ mutant to proliferate on pear fruit and cause symptom development (Fig. 5B; data not shown). Though the $r c s C_{Y p-E c}$ also restored the ability of the $\operatorname{rcs} C$ mutant to proliferate
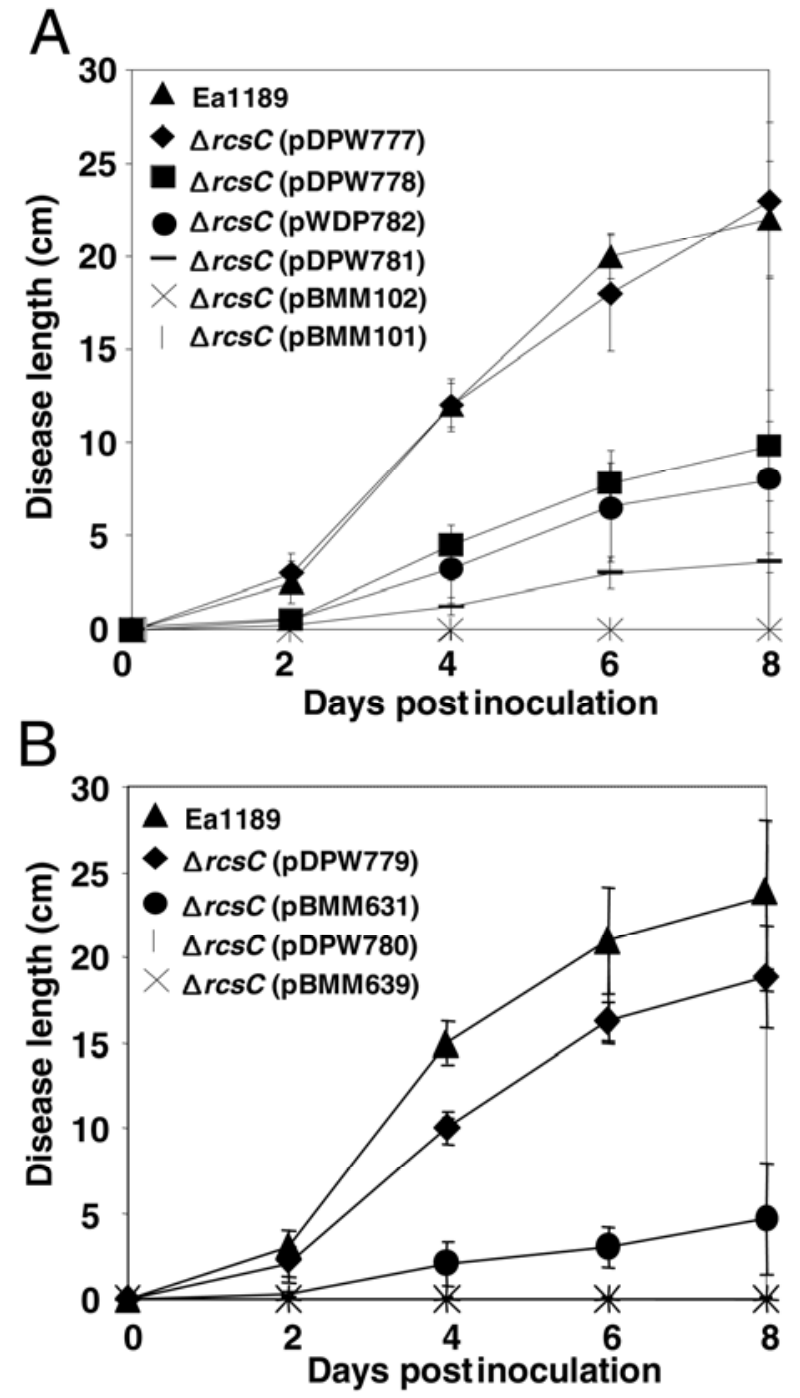

Fig. 4. Disease severity of bacterial strains on 'Gala' apple shoots. A, Disease severity of wild type (WT), $\operatorname{rcs} C$ mutant, and complementation strains on apple shoots. B, Disease severity of WT and $\operatorname{rcs} C$ mutants harboring chimeric constructs. Length of disease progress was measured at 2, 4, 6, and 8 days postinoculation. Similar results were obtained in repeated independent experiments. pBMM101: vector; pBMM102: $r c s C_{E c}$; pDPW777: $r c s C_{E a}$; pWDP778: $r c s C_{Y p}$; pWDP781: $r c s C_{S e} ;$ pWDP782: $r c s C_{P s} ;$ pWDP779: $r c s C_{E a-E c}$; pWDP780: $r c s C_{E c-E a}$; pBMM631: $r c s C_{Y p-E c}$; and pBMM639: $r c s C_{E c-Y p}$. Lines for pBMM101 (I) and pBMM102 (X) were coincided on the X-axis. 
on pear fruit, symptom development was slightly delayed (data not shown) and bacterial population size was reduced by $\approx 10$-fold at 2 and 3 dpi (Fig. 5B). Furthermore, the population size of the $r c s C$ mutant complemented with $r c s C_{E c-E a}$ was significantly reduced by 100 -fold compared with that of the WT strain (Fig. 5B), and disease development was also greatly delayed. However, $\mathrm{RcsC}_{E c-Y p}$ did not support bacterial growth and disease development (Fig. 5B). These results suggested that the $r c s C$ sensor domain might greatly contribute to virulence and survival of E. amylovora on immature pear fruit, and that the histidine kinase and receiver domains were also involved in these activities.

\section{DISCUSSION}

Several genetic factors have been reported to be involved in the regulation of amylovoran, a major virulence factor in Erwinia amylovora $(23,31)$. One of the major and direct regulators of amylovoran production is the Rcs phosphorelay system. We have previously demonstrated that mutations in a single $r c s$ gene results in its inability to cause disease. In particular, the $\operatorname{rcs} C$ mutant yields interesting phenotypes as it produces a high level of amylovoran in vitro but the mutant remains nonpathogenic on host plants. In this study, we have further characterized the $\mathrm{RcsC}$ sensor kinase and its homologs from several enteric bacteria. Our results provide strong evidence that the RcsC kinase has both phosphatase and kinase activities, and these activities play a major role in its ability to control amylovoran production in vitro and disease development in vivo. Our results have also demonstrated that only the chimeric protein $\mathrm{Rcs}_{E c-E a}$ could complement in vitro amylovoran production in the $\operatorname{rcs} C$ mutant, whereas several homologs and $\mathrm{RcsC}_{Y p-E c}$ and $\mathrm{Rcs}_{E a-E c}$ chimeric constructs could partially complement virulence of the $r c s C$ mutant on apple shoots. These results indicate that the sensor domain of RcsC may be important in regulating bacterial virulence, whereas the histidine kinase and receiver domains of Erwinia $\mathrm{RcsC}$ may be essential for suppressing amylovoran production in vitro. Moreover, these findings also suggest that an unknown in planta signal may be involved in this process, and that the level of disease resistance or susceptibility in the plant host may also play a role in this interaction.

It has been shown that amylovoran biosynthesis in the $E$. amylovora WT strain is induced in planta and the $\mathrm{RcsC}$ sensor kinase is required for this process (23). Expression of the ams $G$ gene is lower in the $\operatorname{rcs} C$ mutant than in the WT strain, thus suggesting that $\mathrm{RcsC}$ might possess net kinase activity during plant infection (23). However, under normal growth either in LB or minimum media, expression of the ams $G$ gene (and, thus, amylovoran production) is higher in the $\operatorname{rcs} C$ mutant than in the WT strain. This suggests that $\mathrm{RcsC}$ with net phosphatase activity acts as a suppressor of both ams gene expression and amylovoran production (23). It has also been reported that RcsC in E. coli has a dual function in transferring a phosphoryl group to RcsB (as kinase) or by removing a phosphate from RcsB, in which it acts as a phosphatase (17). However, it remains unclear how RcsC has different functions in planta and in vitro. One possibility is that the kinase/phosphatase ratio may change for the RcsC protein during plant infection. Plant infection may activate some unknown mechanism to suppress phosphatase activity of the $\mathrm{RcsC}$ protein in planta so that the kinase activity of the RcsC protein becomes dominant. It is also possible that, during plant infection, the phosphoryl group transfer reactions from RcsC to $\mathrm{RcsD}$ and then RcsB may be much stronger than the reversible reactions. Host signals that trigger the phoshoprelay reactions may play a role in the function change of the $\mathrm{Rcs} C$ protein by continuously activating this system (pressure), which is lacking in the in vitro condition. Evidence to prove these hypotheses may help elucidate the nature of such in planta signals.
Our results further confirm the net phosphatase activity of $\mathrm{RcsC}$ sensor kinase in the liquid medium as it reduces amylovoran production in $g r r S$, grrA, envZ, ompR, and hns mutants. These results also indicate that $\mathrm{RcsC}$ 's role in amylovoran biosynthesis is independent of the signaling pathways controlled by GrrS/GrrA, EnvZ/OmpR, and H-NS, and it is more direct. This is consistent with the hypothesis that the Rcs phosphorelay system is the most downstream pathway in the regulation of amylovoran biosynthesis in E. amylovora. It has been proposed that the phosphatase activity of $\mathrm{RcsC}$ is mainly dependent on the phosphoreceiver domain. Point mutation studies have demonstrated that the $\mathrm{RcsC}$ phosphatase activity requires presence of D859 in the phosphoreceiver domain but it is highly independent of the presence of $\mathrm{H} 463$ in the histidine kinase domain in E. coli (5). Our results partially support this hypothesis by revealing that only the chimeric protein $\mathrm{RcsC}_{E c-E a}$ containing the phospho-
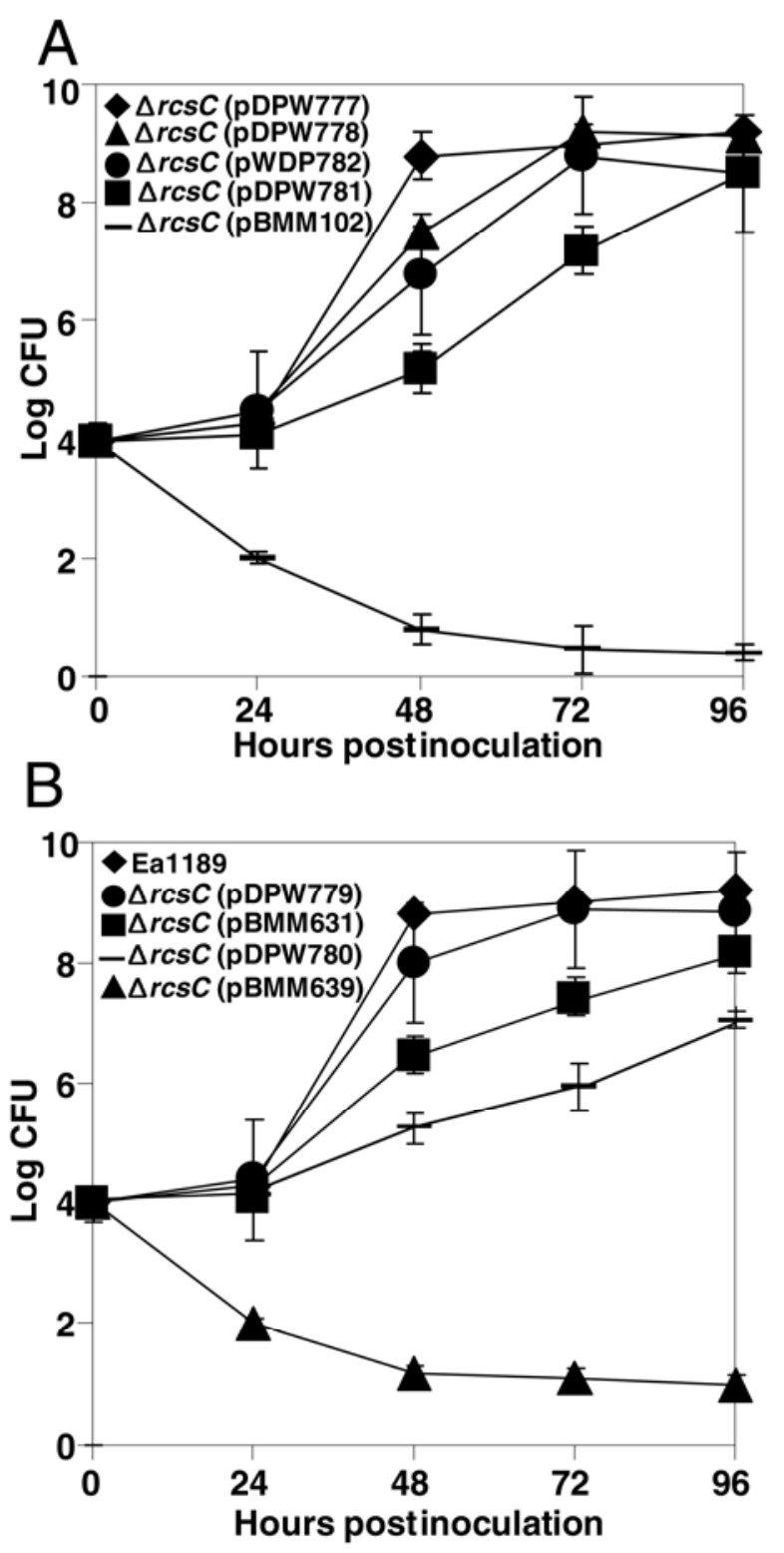

Fig. 5. Bacterial growth of Erwinia amylovora wild type, $\operatorname{rcs} C$ mutant, and complementation strains of the $r c s C$ mutant on immature pear fruit. $\mathbf{A}, r c s C$ mutant complemented with $r c s C$ homologs. B, $\operatorname{rcs} C$ mutant complemented with chimeric constructs. Growth of bacterial strains was monitored at $0,1,2$, 3 , and 4 days postinoculation. Similar results were obtained in repeated independent experiments. pBMM102: $r c s C_{E c}$; pDPW777: $r c s C_{E a}$; pWDP778: $r c s C_{Y p} ; \quad$ pWDP781: $\quad r c s C_{S e}$; pWDP782: $\quad r c s C_{P s} ;$ pWDP779: $r c s C_{E a-E c}$; pWDP780: $r c s C_{E c-E a}$; pBMM631: $r c s C_{Y p-E c}$; and pBMM639: $r c s C_{E c-Y p}$. 
receiver domain of E. amylovora $\mathrm{RcsC}$ fully restores amylovoran production in $\operatorname{rcs} C$ mutant. These results have also demonstrated that all RcsC homologs cannot complement amylovoran production in the $r \operatorname{cs} C$ mutant, suggesting low functional conservation in the phosphoreceiver domain between E. amylovoran and other homologs, and pointing to different net phosphatase activities or even net kinase activities in vitro. These results further confirm previous reports of a different net kinase activity for the $\mathrm{RcsC}$ proteins of various sources (i.e., different kinase/phophatase ratio) (13).

Although RcsC from Yersinia, Pantoea, and Salmonella spp. were not capable of complementing amylovoran production in vitro, they partially restored bacterial virulence in the Erwinia $r c s C$ mutant in planta. Knowledge of how RcsC homologs influencing amylovoran production in vivo is hindered by our inability to measure amylovoran production under these conditions due to technical difficulties. In the immature pear fruit assay, similar amounts of ooze production were observed in both the WT and those strains complemented by RcsC from Yersinia, Pantoea, and Salmonella spp., thus suggesting that they might synthesize similar levels of amylovoran in vivo (data not shown). Similar results were also observed between two different WT strains. Ea273 produced $>10$-fold higher levels of amylovoran than those produced by Ea1189 in vitro, whereas the two strains produced similar amounts of amylovoran and caused similar disease severity on immature pear fruit (24).

It is likely that, upon receiving signals from plants, all $\mathrm{RcsC}$ homologs may act as kinases to promote expression of ams genes and amylovoran production. It is also possible that the phosphoreceiver domains of $E$. amylovora $\mathrm{RcsC}$ homologs behave differently under in vitro and in vivo conditions (i.e., different kinase/phosphatase ratio) (13). Interestingly, we have found that homologs from pathogenic strains (plant or human and animals) could rescue the virulence phenotype of the $\operatorname{rcs} C$ mutant on apple shoots. However, the homolog of a nonpathogenic strain (E. coli $\mathrm{K}-12$ ) could not do so, thus suggesting that there may be some unknown mechanism involved in this process.

Several domain-swapping studies have demonstrated the essential role of the sensor domain for sensing signals $(10,13,14)$. In $E$. coli, the KdpD sensor kinase regulates expression of the kdpFABC operon that encodes the high-affinity $\mathrm{K}^{+}$transport system. Domain-swapping studies using KdpD homologs from $E$. coli, $S$. enterica, Agrobacterium tumefaciens, and $P$. aeruginosa have demonstrated that full responses to salt stress are only achieved with chimerical constructs containing the E. coli KdpD sensor domain (10). The functional variability of the sensor domain has also been observed within a single bacterial species (14). In Streptococcus pneumoniae, the ComD sensor kinase responds to pheromone competence stimulating peptide and phosphorylates the response regulator ComC (14). In 17 sequenced S. pneumoniae strains, ComD had four types of divergent sequences in its first 70 residues. Further studies have shown that these different sensor domains appear to sense two different types of signal peptides (14). Similarly, a chimeric protein composed of the sensor domain of E. coli $\mathrm{K}-12 \mathrm{RcsC}$ and the histidine kinase and receiver domains of $Y$. pestis are capable of fully restoring biofilm formation in E. coli (13). Our results have shown that the sensor domain of $\mathrm{RcsC}_{E p} / \mathrm{RcsC}_{Y p}$ is required for $\operatorname{RcsC}_{E c}$ to be able to complement the $\operatorname{rcs} C$ mutant of $E$. amylovora to cause disease. Because E. coli $\mathrm{K}-12$ is not a pathogen, our results suggest that the sensor domain of RcsC may have evolved to sense specific host signals. Although this host signal remains unknown, it is likely that this signal may be common among animal and plant hosts. Further studies are needed to identify such signals that may provide opportunities to develop effective disease control measures.

Taken together, our results indicate the existence of functional modularity within the RcsC sensor kinase. The sensor domain of
$\mathrm{RcsC}$ may be important for regulating bacterial virulence, whereas the histidine kinase and receiver domains of Erwinia RcsC may be essential for amylovoran production in vitro by acting as phosphatase. Due to current technical difficulties, future work pursuing gene expression, especially in vivo gene expression on apple, would further elucidate the functionality of these genes or various domains. Future studies will also focus on identifying sequences or residues in the sensor domain that are involved in the regulation of bacterial virulence and those signals that are sensed by RcsC during host infection.

\section{ACKNOWLEDGMENTS}

We thank D. Clarke for providing cloning vector and several $r c s C$ constructs and J. M. Slauch and X. Li for providing genomic DNA from Salmonella enteric and Yersinia pestis, respectively. This project was supported by the Agriculture and Food Research Initiative Competitive Grants Program grant no. 2010-65110-20497 from the United States Department of Agriculture (USDA) National Institute of Food and Agriculture to Y. F. Zhao and USDA-SCRI grant AG 2009-51181-06023 to S. S. Korban.

\section{LITERATURE CITED}

1. Altschul, S. F., Madden, T. L., Schaffer, A. A., Zhang, J., Zhang, Z., Miller, W., and Lipman, D. J. 1997. Gapped BLAST and PSI-BLAST: A new generation of protein database search programs. Nucleic Acids Res. 25:3389-3402.

2. Bellemann, P., Bereswill, S., Berger, S., and Geider, K. 1994. Visualization of capsule formation by Erwinia amylovora and assays to determine amylovoran synthesis. Int. J. Biol. Macromol. 16:290-296.

3. Bellemann, P., and Geider, K. 1992. Localization of transposon insertions in pathogenicity mutants of Erwinia amylovora and their biochemical characterization. J. Gen. Microbiol. 138:931-940.

4. Burse, A., Weingart, H., and Ullrich, M. S. 2004. NorM, an Erwinia amylovora multidrug efflux pump involved in in vitro competition with other epiphytic bacteria. Appl. Environ. Microbiol. 70:693-703.

5. Clarke, D. J., Joyce, S. A., Toutain, C. M., Jacq, A., and Holland, I. B. 2002. Genetic analysis of the RcsC sensor kinase from Escherichia coli K-12. J. Bacteriol. 184:1204-1208.

6. Detweiler, C. S., Monack, D. M., Brodsky, I. E., Mathew, H., and Falkow, S. 2003. virK, somA and $r c s C$ are important for systemic Salmonella enterica serovar Typhimurium infection and cationic peptide resistance. Mol. Microbiol. 48:385-400.

7. Erickson, K. D., and Detweiler, C. S. 2006. The Rcs phosphorelay system is specific to enteric pathogens/commensals and activates ydeI, a gene important for persistent Salmonella infection of mice. Mol. Microbiol. 62:883-894.

8. Ferrieres, L., and Clarke, D. J. 2003. The RcsC sensor kinase is required for normal biofilm formation in Escherichia coli K-12 and controls the expression of a regulon in response to growth on a solid surface. Mol. Microbiol. 50:1665-1682.

9. Fredericks, C. E., Shibata, S., Aizawa, S., Reimann, S. A., and Wolfe, A. J. 2006. Acetyl phosphate-sensitive regulation of flagellar biogenesis and capsular biosynthesis depends on the Rcs phosphorelay. Mol. Microbiol. 61:734-747.

10. Heermann, R., Lippert, M., and Jung, K. 2009. Domain swapping reveals that the N-terminal domain of the sensor kinase KdpD in Escherichia coli is important for signaling. BMC Microbiol. 9:1471-2180.

11. Hinchliffe, S. J., Howard, S. L., Huang, Y. H., Clarke, D. J., and Wren, B. W. 2008. The importance of the Rcs phosphorelay in the survival and pathogenesis of the enteropathogenic yersiniae. Microbiology 154:11171131 .

12. Huang, Y. H., Ferrieres, L., and Clarke, D. J. 2006. The role of the Rcs phosphorelay in Enterobacteriaceae. Res. Microbiol. 157:206-212.

13. Huang, Y. H, Ferrières, L., and Clarke, D. J. 2009. Comparative functional analysis of the RcsC sensor kinase from different Enterobacteriaceae. FEMS Microbiol. Lett. 19(7):248-254.

14. Iannelli, F., Oggioni, M. R., and Pozzi, G. 2005. Sensor domain of histidine kinase ComD confers competence pherotype specificity in Streptococcus pneumoniae. FEMS Microbiol. Lett. 252:321-326.

15. Kelm, O., Kiecker, C., Geider, K., and Bernhard, F. 1997. Interaction of the regulator proteins RcsA and RcsB with the promoter of the operon for amylovoran biosynthesis in Erwinia amylovora. Mol. Gen. Genet. 256:72-83.

16. Koczan J. M, McGrath M. J., Zhao Y., and Sundin G. W. 2009. Contribution of the Erwinia amylovora exopolysaccharides amylovoran 
and levan to biofilm formation: Implications in pathogenesis. Phytopathology 99:1237-1244

17. Majdalani, N., and Gottesman, S. 2005. The Res phosphorelay: A complex signal transduction system. Annu. Rev. Microbiol. 59:379-405.

18. Minogue, T. D., Carlier, A., Koutsoudis, M. D., and Bodman S. B. 2005. The cell density-dependent expression of stewartan exopolysaccharide in Pantoea stewartii ssp. stewartii is a function of EsaR-mediated repression of the $\operatorname{rcs} A$ gene. Mol. Microbiol. 56:189-203.

19. Nimtz, M., Mort, A., Domke, T., Wray, V., Zhang, Y., Qiu, F., Coplin, D., and Geider, K. 1996. Structure of amylovoran, the capsular exopolysaccharide from the fire blight pathogen Erwinia amylovora. Carbohydr. Res. 287:59-76.

20. Sambrook, J., and Russel, D. W. 2001. Molecular Cloning: A Laboratory Manual. Cold Spring Harbor Laboratory Press, Cold Spring Harbor, NY.

21. Sjulin, T. M., and Beer, S. V. 1978. Mechanism of wilt induction by amylovoran in Cotoneaster shoots and its relation to wilting of shoots infected by Erwinia amylovora. Phytopathology 68:89-94.

22. Vanneste, J. L. 2000. Fire Blight: The Disease and Its Causative Agent, Erwinia amylovora. CABI Publishing, New York

23. Wang, D., Korban, S. S., and Zhao, Y. F. 2009. The Res phosphorelay system is essential for pathogenicity in Erwinia amylovora. Mol. Plant Pathol. 10:277-290.

24. Wang, D., Korban, S. S., and Zhao, Y. F. 2010. Molecular signature of differential virulence in natural isolates of Erwinia amylovora. Phytopathology 100:192-198.
25. Wang, Q., Zhao, Y., McClelland, M., and Harshey, R. M. 2007. The RcsCDB signaling system and swarming motility in Salmonella enterica serovar typhimurium: Dual regulation of flagellar and SPI-2 virulence genes. J. Bacteriol. 189:8447-8457.

26. Wehland, M., Kiecker, C., Coplin, D. L., Kelm, O., Saenger, W., and Bernhard, F. 1999. Identification of an RcsA/RcsB recognition motif in the promoters of exopolysaccharide biosynthetic operons from Erwinia amylovora and Pantoea stewartii subspecies stewartii. J. Biol. Chem. 274:3300-3307.

27. Zhao, Y. F., Blumer, S. E., and Sundin, G. W. 2005. Identification of Erwinia amylovora genes induced during infection of immature pear tissue. J. Bacteriol. 187:8088-8103.

28. Zhao, Y. F., He, S. Y., and Sundin, G. W. 2006. The Erwinia amylovora avrRpt $2_{E A}$ gene contributes to virulence on pear and AvrRpt2EA is recognized by Arabidopsis RPS2 when expressed in Pseudomonas syringae. Mol. Plant-Microbe Interact. 19:644-654.

29. Zhao, Y. F., and Sundin, G. W. 2008. A simple genetic tool for studying gene function and signal transduction of Erwinia amylovora. Acta Hortic. 793:71-178.

30. Zhao, Y. F., Sundin, G. W., and Wang, D. 2009. Construction and analysis of pathogenicity island deletion mutants of Erwinia amylovora. Can. J. Microbiol. 55:457-464.

31. Zhao, Y. F., Wang, D., Nakka, S., Sundin, G. W., and Korban, S. S. 2009. Systems level analysis of two-component signal transduction systems in Erwinia amylovora: Role in virulence, regulation of amylovoran biosynthesis and swarming motility. BMC Genomics 10:245 